The Astrophysical JouRnal, 507:384-386, 1998 November 1

(C) 1998. The American Astronomical Society. All rights reserved. Printed in U.S.A.

\title{
RAPID FLUCTUATIONS OF WATER MASER EMISSION IN VY CANIS MAJORIS
}

\author{
Xing Wu Zheng, ${ }^{1}$ Eugenio Scalise, JR., ${ }^{2}$ and Fu Han ${ }^{3}$ \\ Received 1997 March 19; accepted 1998 May 11
}

\begin{abstract}
We report the observational results of short timescale monitoring of the $22 \mathrm{GHz}$ water maser emission in VY CMa. A quasi-sinusoidal fluctuation has been detected with the relative flux intensity change of $20 \%-25 \%$ and a period of 10.3 day for two dominant features. This detected variability appears to be superimposed on the normal maser lines. We cannot easily explain the rapid fluctuation with the variation of the radiative input or the strong interstellar scintillation along the line of sight. The variation may be caused by the periodic shock.

Subject headings: circumstellar matter — masers - stars: individual (VY Canis Majoris)
\end{abstract}

\section{INTRODUCTION}

VY CMa is a well-known M5e irregular variable supergiant star immersed in a small reflection nebula and located at the interface of a large molecular cloud and the $\mathrm{H}$ II region S310 (Lada \& Reid 1978). In the infrared, at $20 \mu \mathrm{m}$, it is one of the brightest sources in the sky (Hyland et al. 1969) and at radio wavelengths it displays strong maser emission from $\mathrm{OH}, \mathrm{H}_{2} \mathrm{O}$, and $\mathrm{SiO}$ maser molecules (Herbig 1969, 1972). In recent years a high number of rotational transitions, including the microwave transition of ${ }^{28} \mathrm{SiO} v=4$, $J=5-4$ transition and the rotational lines of the rare isotope ${ }^{29} \mathrm{SiO}$, have been detected with the IRAM radio telescope (Cernicharo et al. 1992). Moreover, five new water maser transitions $\left(4_{4,0}-5_{3,3}\right.$ at $96 \mathrm{GHz} ; 5_{5,0}-6_{4,3}$ at 232 $\mathrm{GHz} ; 7_{7,0}-8_{6,3}$ at $263.5 \mathrm{GHz} ; 1_{2,9}-9_{3,6}$ at $321 \mathrm{GHz}$; and $5_{1,5}-4_{2,2}$ at $325 \mathrm{GHz}$ ) have been observed (Menten \& Melnick 1989, 1991; Yates 1992. The abundant lines from the source allow many authors to infer the structure of the envelope and the conditions of maser excitation. Comparing the line profiles of the well-studied $22 \mathrm{GHz}$ water maser, the (sub)millimeter $\mathrm{H}_{2} \mathrm{O}$ emission, and a high number of SiO masers, Menten \& Melnick (1991) concluded that the vibrationally excited $\mathrm{H}_{2} \mathrm{O}$ and $\mathrm{SiO}$ masers probably arise from a region with infalling motions of matter. Based on the clumps of relatively high velocity detected only at the $v=3$, $J=4-3$, and $v=4, J=5-4 \mathrm{SiO}$ lines, Cernicharo et al. (1992) proposed a rotating disk model since the highest rotational velocity is expected in the inner (more excited) regions of the rotating structures. Evidence for a disk and bipolar flow is clearly seen in the MERLIN maps of circumstellar $22 \mathrm{GHz} \mathrm{H}_{2} \mathrm{O}$ masers around VY CMa (Yates et al. 1992).

The maser variability is an important probe in the dynamics of the circumstellar envelope. However, the availability of copious data on maser variability has not led to significant improvement of our understanding of the physics of the circumstellar environment. One of the first attempts to investigate in detail the long-term time variations of several stars was carried out by Cox \& Parker (1979). They have monitored at irregular intervals the maser

\footnotetext{
${ }^{1}$ Department of Astronomy, Nanjing University, 22 Hankow Road, Nanjing 210093, People's Republic of China; xwzheng@nju.edu.cn.

2 Instituo Nacional de Pesquisas Espaciais-Divisão de Astrofisica C.P. 515 12201-970 S. Jose dos Campos S.P., Brazil.

${ }^{3}$ Purple Mountain Observatory, 2 Beijing West Road, Nanjing 210008, People's Republic of China.
}

emission from VY CMa from 1974 September to 1977 May. After analyzing the data they concluded that the proportional changes in intensity of the three main groups of features indicated the existence of a common pump source. Comparing their results with the spectra obtained by Knowles \& Batchelor (1976) they also concluded that in long-term periods some components presented independent variability. Gomez Balboa, \& Lepine (1986) extended their investigation of the variability of the water emission of VY CMa to 1981. They argued that a 350 day period seemed to be present in all three features and was possibly related to the common infrared pumping source. A monitoring observation of four sources of $\mathrm{SiO}$ maser emission was made by Pijpers et al. (1994). They failed to detect the variation in VY CMa.

With the objective of studying very short interval variation (days to hours) of water maser emission at $22 \mathrm{GHz}$, we undertook a monitoring program of 14 sources. Some interesting results of these observations will be presented elsewhere (Scalise, Han, \& Zheng 1998). Here we present the results of the monitoring of the VY CMa emission and report the detection of a 10.3 day periodicity.

\section{OBSERVATIONS}

The monitoring observations of the short time variation of the water maser were carried out in 1993 from August 26 through September using the $13.7 \mathrm{~m}$ telescope at the Qinghai Station of Purple Mountain Observatory (PMOQH). This antenna is located in the Gobi Desert, a very dry and arid region at $3200 \mathrm{~m}$ above the sea level in western China. It is a classic Cassegrain telescope and has a pointing accuracy better than $20^{\prime \prime}$ and at $22 \mathrm{GHz}$ a HPBW of 4.2. The front end operates with a cooled Schottky mixer and a 1.4 GHz FET IF amplifier. The local oscillator was a phase-locked Gunn diode. A high-resolution 1024 channel AOS was employed as a back end, having a channel spacing of $12 \mathrm{kHz}$, or a velocity resolution of $0.16 \mathrm{~km} \mathrm{~s}^{-1}$ at the observed frequency. Observations were made in positionswitching mode and a $120 \mathrm{~K}$ noise diode was used as a second calibrator. To check the stability of the noise diode a number of continuum sources were observed. During the entire observation period the weather conditions at the site were excellent. We estimate that the atmospheric attenuation at zenith during the entire period was about 0.01 . The absolute calibration for flux density was about $20 \%$.

In order to eliminate any gain dependence effect of the radio telescope all the observations were carried out at the 


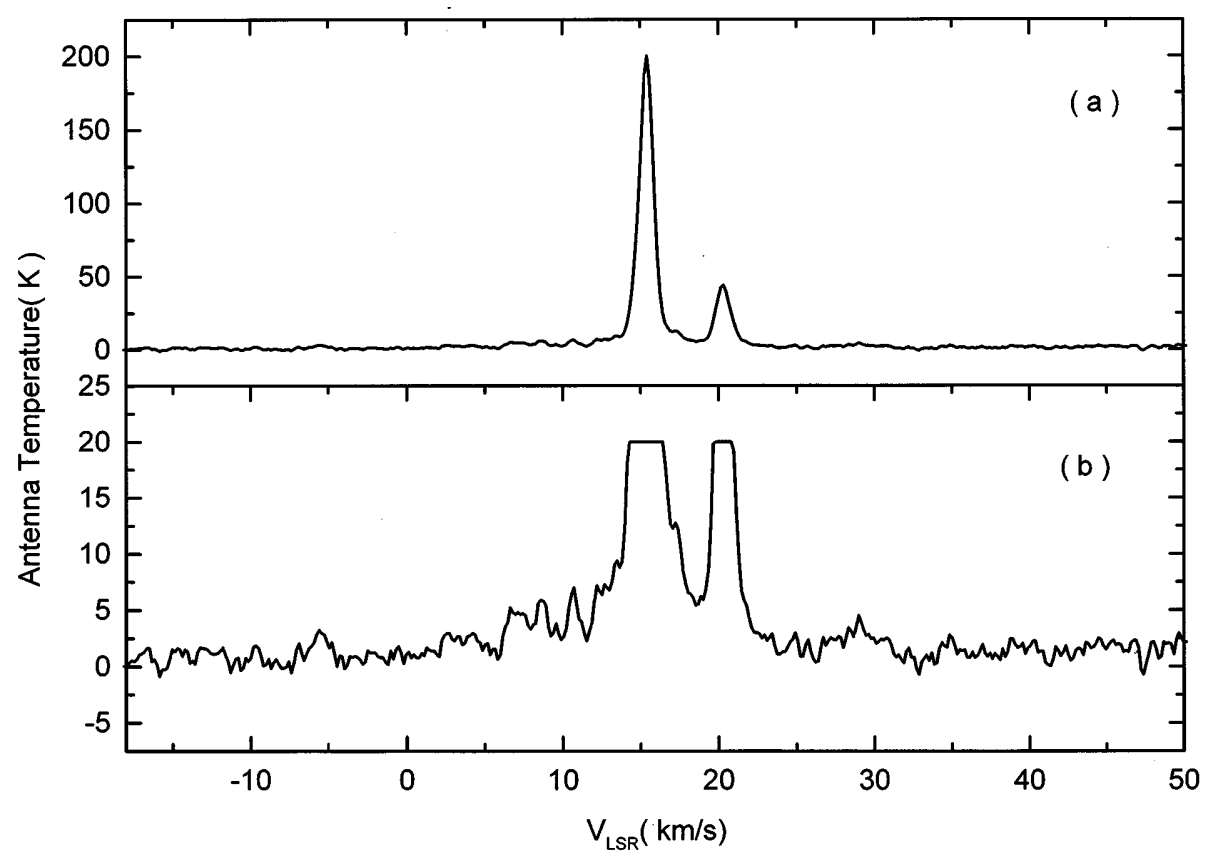

FIG. 1. - Spectrum of the $22 \mathrm{GHz}$ water maser emission from VY CMa. The on-source integration time is $120 \mathrm{~s}(1 \sigma=0.9 \mathrm{~K})$. Velocity resolution of the spectrum is $0.16 \mathrm{~km} \mathrm{~s}^{-1}$. The lower panel displays the spectrum on an expanded scale.

same sidereal time, i.e., at the same parallactic angle and elevation angles. To discriminate the instrumental effects and the real changes in the flux density, the monitoring program included several sources with different spectral features in order to understand the telescope properties.

\section{RESULTS OF THE OBSERVATION}

A spectrum of the water maser emission from the supergiant star VY CMa is given in Figure 1. The rms noise level $(1 \sigma)$ in the plot is about $0.9 \mathrm{~K}$, obtained after $240 \mathrm{~s}$ integration (120 s on-source). At this noise level, we found that the maser lines spread from -10 to $30 \mathrm{~km} \mathrm{~s}^{-1}$. The velocity range is similar to that in spectra observed by many authors (Yates 1992; Menten \& Melnick 1989; Cox \& Parker 1979; Rosen et al. 1978). The central group of features in the velocities from 10 to $25 \mathrm{~km} \mathrm{~s}^{-1}$ appears to consist primarily of two prominent peaks located at 14 and $20 \mathrm{~km} \mathrm{~s}^{-1}$, respectively. The observed characteristics and the ratio of these two components are very similar to those reported by Yates et al. (1992), although Yates quoted 16 and $22 \mathrm{~km} \mathrm{~s}^{-1}$ for these two peaks. The origin of this discrepancy might be caused by the frequency calibrations. These two components are approximately symmetric about the stellar velocity of $17.6 \mathrm{~km} \mathrm{~s}^{-1}$ (Reid \& Dickinson 1976). There are many weaker features around the base of these peaks. Other two groups near 35 to $45 \mathrm{~km} \mathrm{~s}^{-1}$ and -10 to $0 \mathrm{~km} \mathrm{~s}^{-1}$ have varied considerably over the past twenty years. For example, in the spectrum from 1976 September given by Rosen et al. (1978), the component at $-4 \mathrm{~km} \mathrm{~s}^{-1}$ was one of the brightest features, but it has almost disappeared in our spectrum at the $0.9 \mathrm{~K}$ noise level.

Figure 2 shows the flux density variations of these two peaks as function of time scaled by 1 day. The intensity variation amplitudes were about $60 \mathrm{~K}$ for the feature at 14 $\mathrm{km} \mathrm{s}^{-1}$ and $15 \mathrm{~K}$ for the feature at $20 \mathrm{~km} \mathrm{~s}^{-1}$, respectively. The relative changes in the intensity for these two features are approximately the same, about $30 \%$. The fluctuations appear to be sinusoidal with a period of 10.3 days. The variation of the line profile integration also follows the period. The average error for the Gaussian fit to the spectra is about $0.5 \mathrm{~K}$. It should be noted that during our observations more than one scan was obtained on several

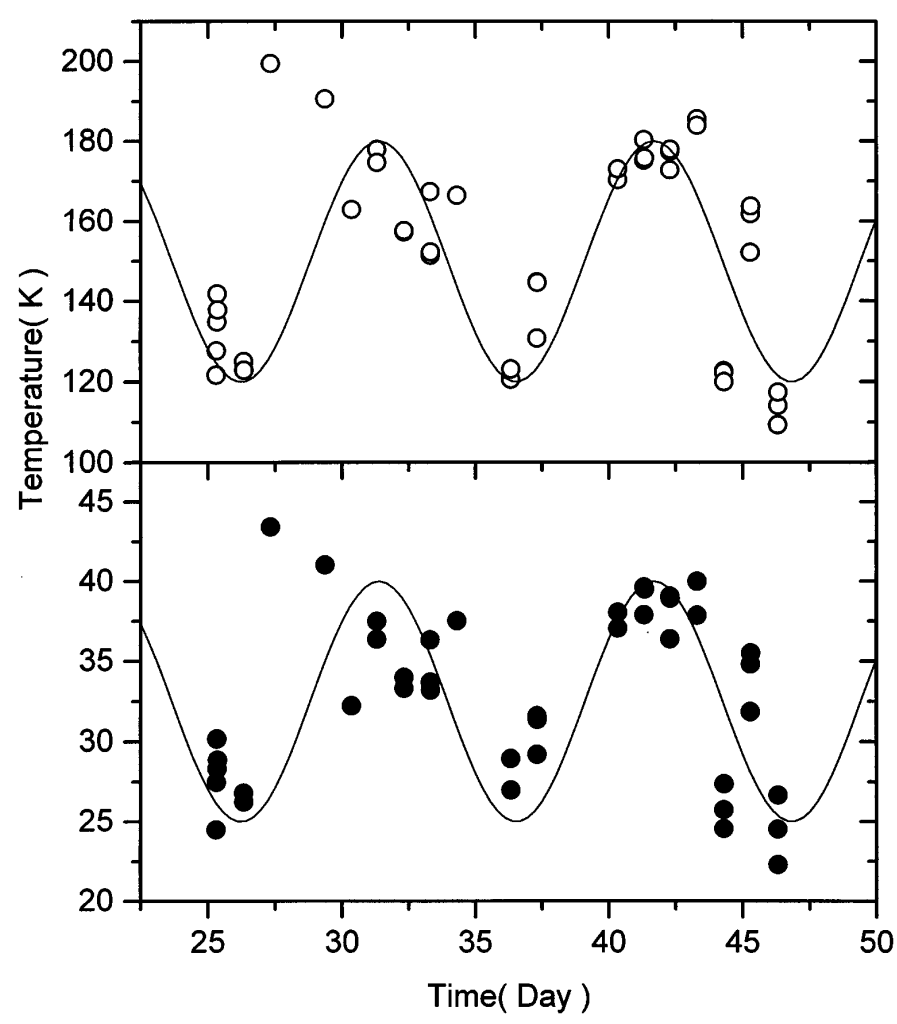

FIG. 2.-Variation curves of the radio flux densities for two peaks at velocities of $14 \mathrm{~km} \mathrm{~s}^{-1}$ (top) and $20 \mathrm{~km} \mathrm{~s}^{-1}$ (bottom), respectively. Flux densities are corrected by atmospheric attenuation, antenna gain variations, and pointing error. The average error for the fitting Gaussian line profiles to the spectra is about $0.5 \mathrm{~K}$. The sinusoid overlaying on the plot is in the same period, 10.3 days, with different amplitudes: $60 \mathrm{~K}$ for the feature at $14 \mathrm{~km} \mathrm{~s}^{-1}$ and $15 \mathrm{~K}$ for one at $20 \mathrm{~km} \mathrm{~s}^{-1}$. The monitoring observations were carried out in 1993 August 26 through September 24. 
occasions. The error of the flux density between different scans was not greater than $20 \mathrm{~K}$ for the feature at $20 \mathrm{~km} \mathrm{~s}^{-1}$ and $5 \mathrm{~K}$ for the feature at $14 \mathrm{~km} \mathrm{~s}^{-1}$. We also examined carefully the shifts of radial velocities for these two components. The systematic shifts of $0.01 \mathrm{~km} \mathrm{~s}^{-1}$ per day are probably caused by the instrumental frequency axis. It is difficult to identify a phase lag between these two curves of variations in Figure 2.

\section{COMMENTS OF THE RESULTS}

It is likely that the apparent maser fluctuations are mostly real and are not caused by instrument effects. The amplitudes of these two sinusoidal curves are different, and instrument effects could not be responsible for such percent variations in different channels. In addition, observations of 13 other masers including $\mathrm{W} 3(\mathrm{OH}), \mathrm{W} 51 \mathrm{~N}$, and WHya done on the same days as part of our short time variation study of the water maser variability, do not reveal any periodic variation (Scalise et al. 1998). The 4.2 primary beam is much larger than the $20^{\prime \prime}$ pointing accuracy, so pointing errors could not cause the apparent variability for the maser structures located in the envelope of the VY CMa supergiant star. The half-power width of these two peaked features are over more than seven channels. Hence there was no undersampling of spectral lines which might have caused the variations. Any polarization effects are not significant because all observations were made at approximately the same sidereal time.

The same period of the flux variation for two features at 14 and $20 \mathrm{~km} \mathrm{~s}^{-1}$ indicates the existence of a common source of the "seed" variation. Approximately the same percent of variation for these two features may come from the same physical factors in different regions. In the absence of other data such as IR and visual observations it is difficult to identify the origin of the maser variability; however, based on our radio observation, we tentatively make the following points.

Three mechanisms could produce the maser variability in the circumstellar envelope of VY CMa: a quasi-sinusoidal input signal, a quasi-sinusoidal pumping rate, or the interstellar scintillation.

If the maser is unsaturated, the maser output would be expected to follow the sinusoidal input variation. It is, however, hard to explain the 10.3 day sinusoidal oscillation of the maser. For variable supergiant stars like VY CMa, the period of oscillations always scales inversely proportional to the square root of the mean density (Cox 1980). The supergiant stars have a characteristic period of about 300 days. Harmonic oscillations near 10 days must be weak. Moreover, it is difficult to explain the linear changes in the two features in terms of the input variability.

Intermolecular collisions are generally thought to be responsible for the pumping mechanism of the water masers in the late-type stars. The changes in the collision rates produced by periodic shock waves could introduce the apparent maser variability. The photosphere acts as a pulsating piston and injects a large-amplitude shock wave into the gas envelope once each pulsation cycle. The inner layers close to the star may be driven outwardly by directed shock fronts and drawn back to the photosphere surface by gravity (Wood 1979; Hill \& Willson 1979). This phenomenon may provide a satisfactory explanation of the quasisinusoidal fluctuation of the intensities of the maser emission. For the saturated maser, the linear and periodically simultaneous variation can be explained by an expanding shell with $3 \mathrm{~km} \mathrm{~s}^{-1}$ velocity at a distance of $10^{16}$ $\mathrm{cm}$.

Interstellar scintillation caused by a scattering medium along the line of sight may play an important role for the explanation of the short time fluctuation of the $22 \mathrm{GHz}$ water masers. For the strongly diffractive scintillation, the correlation timescale is $t_{d} \sim \lambda / 2 \pi \theta_{s} v_{t}$ for a source of angular size $\theta_{s}$, observing wavelength $\lambda$, and a relative transverse velocity $v_{t}$ (Simonetti 1992). For $D=1.5 \mathrm{kpc}$ (Herbig 1969), the diameter of maser spot $d_{s}=1.5 \times 10^{13} \mathrm{~cm}$ (Rosen et al. 1978) and $v_{t}=60 \mathrm{~km} \mathrm{~s}^{-1}$, the diffractive timescale is about $10 \mathrm{~s}$. It is too short compared to our observing time amplitude. The correlation timescale of refractive interstellar scintillation is $t_{r} \sim d_{0} / v_{t}$, where $d_{0}$ is the observed angular size. For the assumption $d_{0}=d_{s}$, the refractive timescale is about 29 days, 3 times longer than our observations. Therefore, the interstellar scintillation is difficult to explain in 10.3 day variations.

Based on the above discussion, we tentatively conclude that the maser variability may be caused by the periodic shock. If the idea is true, the maser emission probably arises from a region close to the star. For the outer layers the matter driven by the shock does not return to its original position before the arrival of the next shock wave and superperiods may be established. If our observing variations are correct short time periodical variability of these two features originated from the same stellar optical or IR radiation in the pumping of the water maser. To better understand whether there is real periodicity present in the short timescale of VY CMa and to provide reasonable analysis for the origin of variation, a monitoring program with much longer duration is needed, with both the infrared and optical data.

We are grateful to the PMOQH observational and technical staff for their assistance during our observations; J. Lü and Z. F. Ling for help in data calibration; H. J. Su and P. S. Chen for useful discussions; and D. R. Xiong for carefully reading the manuscript. The observations were supported by the National Scaling Project and the United Open Radio Astronomy Laboratory in China.

\section{REFERENCES}

Cernicharo, J., Bujarrabal, V., \& Santaren, T. L. 1992, in Astrophysical Masers, ed. A. W. Clegg \& G. E. Nedoluha (Heidelberg: SpringerVerlag), 425

Cox, J. P. 1980, Theory of Stellar Pulsation (Princeton, NJ: Princeton)

Cox, G. G., \& Parker, E. A. 1979, MNRAS, 196, 197

Gomez Balboa, A. M., \& Lepine, J. R. D. 1986, A\&A, 159, 166

Herbig, G. H. 1969, Mem. Soc. Roy. Sci. Liége, Ser. 8, vol 13

.1972, ApJ, 172, 375

Hill, S. J., \& Willson, L. A. 1979, ApJ, 229, 1029

Hyland, A. R., Becklin, E. E., Neugebauer, G., \& Wallerstein, G. 1969, ApJ, 158,619

Knowles, S. H., \& Batchelor, R. A. 1979, MNRAS, 174, 69

Lada, C., \& Reid, M. 1978, ApJ, 219, 95
Menten, K. M., \& Melnick, G. J. 1989, ApJ, 341, L91 1991, ApJ, 377, 647

Pijpers, F. P., Pardo, J. R., \& Bujarrabal, V. 1994, A\&A, 286, 501

Reid, M. J., \& Dickison, D. F. 1976, ApJ, 209, 505

Rosen, B. R., Moran, J. M., Reid, M. J., Walker, R. C., Burke, B. F., Johnson, K. J., \& Spencer, J. H. 1978, ApJ, 222, 132

Scalise, E., Jr., Han, F., \& Zheng, X. W. 1998, in preparation

Simonetti, J. H., Diamond, P. J., Uphoff, J. A., Boboltz, D., \& Dennission, B. 1992, Astrophysical Masers, ed. A. W. Clegg \& G. E. Nedoluha (Heidelberg: Springer-Verlag), 311

Wood, P. R. 1979, ApJ, 227, 220

Yates, J. A. 1992, in Astrophysical Masers, ed. A. W. Clegg \& G. E. Nedoluha (Heidelberg: Springer-Verlag), 415 